\title{
TRABAJO SOCIAL Y SERVICIOS SOCIALES: CONFUSIONES Y DESCONOCIMIENTOS
}

Fernando de Lucas y Murillo de la Cueva*

Uno de los problemas que dibujan el Trabajo Social y los Servicios Sociales radica en la indefinición y confusión sobre algunos de sus contornos. Como es sabido, cuando surge una nueva disciplina social, es natural que ésta se esfuerce en establecer su propio estatuto científico. Este es el caso del Trabajo Social, que como disciplina joven que es, tropieza con dificultades, escollos que poco a poco se van sorteando. De hecho, hay que alabar la tarea admirable realizada por numerosos profesores que están transmitiendo sus conocimientos a los alumnos. Pero todavía queda camino por recorrer.

Con respecto a los Servicios Sociales, los problemas son similares, pero no podamos nombrar a los mismos como ciencia. Son una técnica, una disciplina de estudio, una realidad social íntimamente ligada al Trabajo Social, aunque con su propia personalidad.

En las líneas siguientes, pretendo tratar, brevemente, algunas cuestiones que dificultan la correcta perfilación de ambas realidades, empezando por abordar los problemas relativos a la historia de ambas; la cuestión científica; lo relativo a la especificidad entrambas; el problema terminológico; el conocimiento del Trabajo Social y los Servicios Sociales por los ciudadanos; las mediaciones que impactan en ambos y que, invariablemente generan nuevos problemas e, incluso, necesidades; y el papel de la oferta social. Por último realizaré algunas reflexiones sobre el futuro de Trabajo Social y Servicios Sociales al hilo de las actuales tendencias en el pensamiento.

*Doctor en Ciencias Políticas y Sociología. Profesor Titular de la Universidad Complutense de Madrid. Miembro de la ACMS. 
En primer lugar, cuando se inicia la aproximación al concepto y realidad de cualquier hecho, es indispensable referirse a su propia historia, a la forma en que se ha ido configurando y construyendo a lo largo del tiempo. Pues, bien, en el caso de la historia y precedentes del Trabajo Social y de los Servicios Sociales, nos encontramos ante el problema de que, en ocasiones, se describen aquéllos emparentados en la misma historia. Realmente no es así.

La confusión entre las raíces de la Acción Social, los Servicios Sociales y la historia del Trabajo Social es más habitual de lo que parece. Este no es un asunto baladí. Tiene gran importancia pues, si considerásemos al Trabajo Social -profesión que se configura realmente a partir del siglo XIX-, compartiendo la historia de la Acción Social, sería bien difícil desbrozar los Servicios Sociales del aquél. Porque, según esta visón, llegarían a nuestros días históricamente imbricados.

Juan Luis Vives desarrolló argumentos, trazó perspectivas y propuestas que, sin duda, entroncan con el Trabajo Social, pero su legado no es Trabajo Social. Tampoco la práctica profesional del Trabajo Social es resultado histórico de los Servicios Sociales (realidad anterior en el tiempo), sólo se sirve de ellos y encuentra lugar profesional, a través de la consolidación del sistema público de Servicios Sociales, que es una cosa bien diferente.

$\mathrm{Si}$ este es un grave problema, adquiere mayor relevancia cuando lo contemplamos en el ámbito docente y académico. Aunque la enseñanza de Servicios Sociales en Escuela de Trabajo Social en España, es muy reciente (1983), todavía hay tiempo para no confundir al alumnado que, en el futuro, esperamos se incorpore al ámbito laboral como Trabajadores Sociales, investigadores, docentes, etc., sin perpetuar la confusión que aquí citamos. Si desde el primer curso de la Diplomatura el alumno tropieza con obstáculos a la hora de poder delimitar conceptual e, históricamente el Trabajo Social y los Servicios Sociales, avanzaremos más en nuestra tarea docente.

Así, pues, todos sabemos que Trabajo Social y Servicios Sociales no son la misma cosa, aunque permanezcan muy cercanos. Por eso parece pertinente acordar la distancia (temporal incluso) entrambos: situamos la emergencia efectiva (aunque sus precedentes sean anteriore) de los Servicios Sociales a partir de los siglos XVIXVII, sin embargo fue el siglo XIX, como hemos dicho, el punto de partida para calibrar al Trabajo Social en cuanto profesión.

Los problemas no acaban aquí, pues la misma juventud del Trabajo Social contribuye a complicar su definición. El Trabajo Social es una realidad que pelea por recabar su estatuto científico. Mas su carácter multidimensional y multidisciplinar, requiere la colaboración de otras Ciencias, habitualmente Sociales, que tampoco se pueden distinguir por su pureza científica y objetiva.

El carácter subjetivo de las llamadas Ciencias Sociales, Ciencias del Espíritu y Ciencias Humanas (división que Dilthey estableció entre Gesteswissenschaften y Kulturwissenschaften) brota, principalmente, de la presencia humana en el objeto de estudio. No obstante no es esta razón suficiente para negar el contenido científico 
de cualquier disciplina que lleve el apellido de "social". Max Weber afirmaba certeramente que somos nosotros los más indicados para descubrir los matices subjetivos de toda Acción Social, pues los podemos reconocer en nosotros mismos.

En este caso, el problema se reduce, pero no acaba aquí. El Trabajo Social, y más aún los Servicios Sociales, dotados ambos de ese rasgo multidimensional (más aún el primero, en su investigación e intervención de la familia, los grupos y la comunidad) no pueden desempeñarse sin el concurso de estas otras disciplinas, como acabamos de decir. Así se enriquecen ambas, pero también surgen dificultades.

Otra de las razones por las que los científicos llamados "puros" se resistan a admitir la cientificidad de las disciplinas calificadas de sociales, se halla en su acusación de falta de unidad, de excesiva profusión de paradigmas y la relativa validez de todos ellos. No tratamos aquí iniciar un debate sobre Sociología, Psicología de la Ciencia y los estudios históricos que adquirieron especial empuje tras la Segunda Guerra Mundial tanto en Europa como en Estados Unidos, baste recordar la famosa obra de Thomas S. Khun a propósito de La estructura de las revoluciones científica, en el sentido de la dependencia que toda la ciencia (incluida la llamada pura, natural o exacta) tiene con relación a realidades extra-científicas, que determinan y orientan a la investigación, investigación que ya no podrá ser calificada de neutral y objetiva como antes se hacía.

Por este camino nos introduciríamos en el debate sobre la postmodernidad, sobre la dialéctica entre ciencia moderna y postmoderna, sobre el problema de la verdad y la propia razón emancipadora, cosa que no voy a hacer, aunque me interesa que quede claro que caminamos por senderos sinuosos y poco seguros.Este es un asunto especialmente preocupante en el caso de los Servicios Sociales y, como así creo, en el del Trabajo Social.

Los Servicios Sociales, siendo respuestas técnicas, instrumentos que puede, como así hace, utilizar el Trabajo Social, no son herramientas neutras, por supuesto. Son resultado del esfuerzo de profesionales, de debates políticos, son expresiones del Derecho, pero no poseen la dimensión y propósitos del Trabajo Social. La tarea de los Servicios Sociales no es tanto intervenir en la realidad social, por muy carencial que esta sea, sino que sirven para ser aplicados en esa realidad. Quien, de hecho, interviene, es el Trabajo Social.

Un Servicio Social mal diseñado puede, acaso, ser remodelado en la práctica del Trabajo Social, aunque no es menos cierto que un Servicio Social, esté bien o mal diseñado, si es mal ejecutado pierde su utilidad y genera mayores problemas. A fin de cuentas, la dimensión humana y profesional del Trabajo Social puede, y así parece, ser más relevante.

Porque el Trabajador Social, aunque a veces ocurra, no debe ser un mero funcionario o gestor de servicios, sino que debería guardar un grave compromiso con su profesión pues, a quien va destinada, no lo olvidemos, suele encontrarse en el campo de la pobreza, marginalidad y exclusión social.

En este compromiso, en su tarea sobre la Comunidad, los grupos, familias e individuos, necesita poseer todas las herramientas (conceptuales e instrumentales) 
a punto así como prepararse para poder replantear la pertinencia y reforma de ellas. Así, pues, es difícil tal objetivo si no se parte de un marco estable de estudio, más aún si dejamos al profesional que replantee sus conocimientos y experiencia en el día a día.

No me estoy refiriendo a los sucesivos impactos del psicoanálisis (teoría psicodinámica), del pragmatismo norteamericano, el interaccionismo simbólico, conductismo, teoría de sistemas, teorías críticas, etc., en los ámbitos de actuación del Trabajo Social y, también, sobre los Servicios Sociales. Esto ha ocurrido en el Trabajo Social y en todas las Ciencias Sociales que, con el afloramiento y pugna de, $y$ entre, diversos paradigmas se enriquecen.

De lo que se trata es de crear un substrato homogéneo para el Trabajo Social y para los Servicios Sociales, y este puede alcanzarse en cierta medida, unificando criterios, otorgando mayor rigor conceptual y metodológico a nuestras tareas. En contra de este desideratum, aparecen otros problemas, que contribuyen, a la confusión entre Trabajo Social y Servicios Sociales.

Una ciencia, para serlo, cualquier disciplina mínimamente científica, cualquier actividad científica interesada en intervenir en la realidad social con éxito, necesita adquirir cierta especificidad. Bien lo sabía Emile Durkheim, como se desprende de su obra Las reglas del método sociológico, y su disputa con Gabriel Tarde. Pues bien, conocemos el solapamiento producido entre Trabajo Social y Servicios Sociales, no a partir de la tarea equivocada o errónea de éste o aquél profesional, o de esta o aquélla decisión política o ministerial, sino en el mismo empleo de los Servicios Sociales por parte de los Trabajadores Sociales a partir de la puesta en marcha de la Democracia en nuestro país. Sobre todo desde la aparición de las diversas Leyes Autonómicas del sector, la Ley de Bases del Régimen Local (2 de Abril de 1985) y del Plan Concertado de Prestaciones Básicas de Servicios Sociales en las Corporaciones Locales (1988).

El protagonismo que recabaron el Municipio y la Administración Local en materia de Servicios Sociales, no cabe duda que era necesario, sobre todo cuanto reparamos en la idea de acercar el recurso al cliente. Estas normativas también sirvieron para proveer mayor relevancia y recursos técnicos a los trabajadores sociales. Ahora bien, los efectos de tales medidas sobre el ejercicio del Trabajo Social, fueron también de cierta confusión, en el sentido de que se llegaron a equiparar Servicios Sociales con Trabajo Social. En algún caso, incluso, se han llegado a definir los Servicios Sociales (caso de hispanoamérica y Francia) como "la actividad llevada a cabo por los profesionales Asistentes Sociales".

Así llegamos a otro de los problemas que dificultan la correcta aproximación al Trabajo Social y a los Servicios Sociales, como es el caso de la confusión terminológica, que es especialmente grave en el caso de los segundos. Además, si los Servicios Sociales son, entre otras cosas, resultado de decisiones políticas, si se convierten en un derecho para los ciudadanos, pueden quedar limitados en su contenido y efectividad si no se sabe con claridad en qué consisten y para qué sirven. Más aún cuando esa duda la tiene el propio legislador. 
En primer lugar, en más de una ocasión se equiparan los Servicios Sociales junto con la Acción Social y el Bienestar Social, pero a ellos también se le suman la Asistencia Social y el propio Trabajo Social. El uso que del término Acción Social hacemos en España en Trabajo Social y Servicios Sociales (no referido al concepto madre, el sociológico), parece equipararse a los Servicios Sociales. De hecho, son diversas las leyes autonómicas del sector que así se denominan ("de Acción Social"). En Francia se utiliza este concepto en un sentido similar al de los Servicios Sociales británicos. Cáritas lo usó para potenciar intervenciones promocionales en detrimento de la tradición asistencialista. También se ha empleado en algunas empresas para designar actividades sociales de las mismas.

A pesar de estos otros usos, y de que, incluso, titule una Dirección General de Acción Social de la Administración Pública que no cubre todos los campos de los Servicios Sociales, podemos equiparar la Acción Social a los mismos. Lo que no elimina cierta dificultad para su aproximación, sobre todo para los estudiantes.

A este término se le suma el de Bienestar Social, que, en el caso francés, podemos indentificarlo como Action Social. Bien es cierto que, según la perspectiva francesa, británica (Social Welfare) y española, parece perseguir siempre la consecución de la "calidad de vida", concepto que también aparece en nuestra Constitución (artículo 45,2 con relación al Medio Ambiente, y 129,1, referido a la participación en la Seguridad Social y los organismos públicos). Sobre el concepto de Bienestar Social podemos aceptar que remite a la noción amplia de los Servicios Sociales, es decir: “...el complejo de acciones públicas y privadas que se arbitran con objeto de satisfacer necesidades humanas y oportunidades sociales. En este conjunto de acciones se indentifican los siguentes subsectores funcionales: Sanidad, Educación; Empleo; Urbanismo y vivienda; Prestaciones económicas y; Servicios Sociales en sentido específico o Servicios Sociales personales". Asi los conciben los británicos. Es decir, el concepto de los Servicios Sociales en el sentido amplio.

En suma, tres términos diferentes: Servicios Sociales, Acción Social y Bienestar Social, pueden designar la misma cosa.

Los problemas aumentan cuando entramos a tratar el término Asistencia Social. Históricamente fue creada para superar, política y técnicamente, a la Beneficencia en sus tareas de contenido social. Bien es cierto que su campo material son las necesidades, como es el caso de los Servicios Sociales, pero ella resume una serie de contenidos que no cuadran con los Servicios Sociales:

1-Prestaciones destinadas a personas necesitadas;

2-Prestaciones concedidas con carácter discrecional;

3-Prestaciones financiadas con fondos de origen fiscal (Asistencia Social Pública).

Ahora bien, a partir de la Constitución de 1978, el problema se incrementó. La combinación de una cierta indeterminación, o claridad, en el concepto de la práctica asistencialista y de los Servicios Sociales, se hizo ley. ¿Por qué?, porque los juristas, administradores y políticos variaron el sentido de ésta conectándola con los Servicios Sociales. 
De esta manera, la Asistencia Social, en lugar de ser una forma institucional de distribución de bienes y servicios, convirtió los mismos en su objeto material. Más aún cuando la misma Constitución permite que la Asistencia Social pueda ser asumida dentro de las competencias de las Comunidades Autónomas. Esto lleva a que, según qué leyes autonómicas (como la antigua madrileña) y, según qué párrafos (algo que ocurre también en la Constitución) se confunda Asistencia Social con Servicios Sociales. Mientras, el Estado restringe sus competencias sobre tal asunto y las Comunidades Autónomas las amplían. A la Acción Social se le suma la Asistencia Social para complicar más el panorama terminológico dentro de nuestra Carta Magna (no olvidemos, como nos recuerda Demetrio Casado, que en la elaboración de la Carta Magna, el diputado Martín-Retortillo logró eliminar la palabra "Beneficencia" de los primeros borradores. El problema es que se eliminó la palabra, pero no la institución).

Así, pues, con el excesivo empleo de sentidos para explicar la Asistencia Social se la comprende como: socorro de pobres, forma institucional de distribución de dicha ayuda, técnica prestacional de Servicios Sociales y campo material de distribución de bienes y servicios. La confusión que se produce en la población, en los especialistas, juristas, políticos, docentes, alumnos, etc., no extraña. $\mathrm{M}^{\mathrm{a}}$ Eugenia Zabarte ha llegado a decir, con razón, que no debe utilizarse más este término, salvo en su vertiente histórica. De esta manera se evitarían confusiones, y lo que es más grave, la creación de una parcela de poder jurídico-político-administrativo que no debe tener cabida en el panorama de la intervención social.

Tampoco se le escapa a nadie que los Asistentes Sociales eran los especialistas que intervenían directamente en la Asistencia Social. En la actualidad todavía se utiliza, indistintamente, la locución "Asistente Social" y la de "Trabajador Social". Fuera de nostalgias pasadas, creo oportuno utilizar la segunda, más aún para los docentes de las Escuelas de Trabajo Social para evitar más confusiones que, como hemos visto, pueden llevar a entroncar al Trabajo Social, los Servicios Sociales, la Acción Social y el Bienestar Social con la Asistencia Social.

No nos sorprende entonces que los Servicios Sociales y el Trabajo Social sean realidades no convenientemente conocidas o, lo que es peor, equivocadamente consideradas por profesionales y especialistas de otros campos. Más nos preocupa que los Servicios Sociales, como prestaciones técnicas y como instrumentos del Trabajo Social, sean auténticos desconocidos por parte de los ciudadanos (que deberían colaborar para su mejor diseño) y mal comprendidos por los clientes de los

mismos.

Si examinamos el campo de las Prestaciones Básicas de los Servicios Sociales, del Primer Nivel o puerta de acceso al sistema de Servicios Sociales, nos encontramos con que las prestaciones de Información, Valoración y Orientación, pueden quedar mediatizadas, más aún cuando los recursos técnicos y humanos no son suficientes. Según la doctrina, un Centro de Servicios Sociales es una estructura integrada de recursos humanos, técnicos, financieros y materiales a través de la cual se gestionan 
los correspondientes programas para hacer efectivas, en un ámbito comunitario determinado, las prestaciones básicas mencionadas, así como el fomento del voluntariado y la cooperación social, todo ello dentro de ese Primer Nivel de los Servicios Sociales que convierte, como hemos dicho, a estas estructuras en la "puerta de entrada" al sistema de Servicios Sociales.

Bien, si consideramos válida tal definición, apreciamos que esta construcción, en la teoría, pretende acercar el recurso al ciudadano, pues es en el ámbito local, como se desprende de nuestro Texto Constitucional y en las mismas Leyes de Servicios Sociales de las diferentes Comunidades Autónomas, donde se aprecian mejor las demandas, conflictos y problemas de la población. Pero, a su vez, y haciendo un poco de etnometodología ligera, podríamos preguntar, y escuchar, cómo definen los usuarios y la población a los Centros de Servicios Sociales, a los mismos Servicios Sociales, al Trabajo Social.

El problema es que, un servicio que no se conoce difícilmente puede usarse, y es más preocupante el hecho que, cuando la información es deficiente e incompleta, conduce a expectativas falsas y, consiguientemente, frustrantes. Esa persona desinformada, en su quehacer cotidiano, tiene más deberes que derechos, y un incremento en sus carencias cuando la información es incompleta.

Bien es cierto que los profesionales de estos centros (trabajadores sociales, psicólogos, sociólogos, animadores socioculturales, etc.) pueden padecer un exceso de demanda de todo tipo. En la Comunidad Autónoma de Madrid tienen que atender no sólo a los clientes para los que originalmente se diseñó tal estructura, sino también a aluviones de estudiantes y diplomados en Trabajo Social que están desarrollando sus trabajos y proyectos de investigación: estudiantes, diplomados, licenciados y profesionales de psicología, sociología, enfermería, graduados sociales, etc., que desean información y datos para sus estudios y trabajos. La paradoja resultante es llamativa: casi nadie conoce estos Centros y los recursos y programas de que disponen, pero no es extraño que quienes allí trabajan estén totalmente saturados en su tarea cotidiana. Tal situación provoca que algunos trabajadores sociales opinen en contra de una mayor divulgación de este tipo de servicios pues apenas pueden atender a la actual demanda.

Son conscientes también de que algunos de estos Centros no están equipados convenientemente. Los usuarios se encuentran, en ocasiones, que tienen que manifestar sus problemas en estancias ruidosas, con continuos trasiegos de personas desconocidas. Él, o la trabajadora social, se encuentran, a veces, abrumados tras un mar de papeles y expedientes. En estos casos, la Información y Orientación quedan mediatizadas, dificultadas. No en vano el medio es el mensaje, por lo que se coarta la relación entre la oferta y la demanda.

El entrevistador debe, en este orden de cosas, recabar información del usuario para percibir su situación con claridad para poder proveerle el recurso más conveniente. La comunicación, proceso interactivo tan complejo, aparece llena de ruidos (en todas sus facetas). ¿Cómo esperar, entonces, a pesar de la preparación 
profesional y la implicación en su tarea de los trabajadores sociales, que puedan desarrollar una labor conveniente cuando los recursos y equipamientos de que disponen se lo impiden?.

Estamos ante una situación en la que las necesidades, objeto material de los Servicios Sociales, pueden generarse desde el mismo sistema. Lo que nos lleva a una nueva reflexión sobre el papel de los trabajadores sociales y los Servicios Sociales.

En este caso me refiero a la mediación profesional que, los trabajadores sociales, han llegado a establecer en el campo material de su labor.

El Trabajo Social y los Servicios Sociales, no son realidades construidas únicamente para situaciones de pobreza extrema y de marginación social. Es claro que, en un vistazo apresurado de las diversas leyes autonómicas del sector, ubican su objeto material en el campo de la marginación social, por eso se comprende, más aún, que se asignen a los Servicios Sociales, con cierta prioridad, el objetivo de prevención.

Los trabajadores sociales, los profesionales españoles centrados en este campo, es cierto que han prestado un interés intelectual, ideológico y gremial sobre los Servicios Sociales como prestaciones técnicas. Pero, como la profesora Guillén afirma, al rechazar las viejas fórmulas de auxilio material de indigentes -caso de la Asistencia Social en una de sus acepciones-, no se propugnaron acciones para conseguir, por ejemplo, subsidios de garantía de rentas mínimas. No hay que olvidar que en el perfil de la demanda social, sigue pesando, con insistente y permanente fuerza, la pobreza en todas sus dimensiones. No podemos olvidar que buena parte de las leyes del sector fueron reacias a incluir el área de "pobreza y marginalidad genéricas y desorganización comunitaria" por el entronque que los pobres poseían desde siempre con la institución benéfica.

El problema se incrementa cuando la captación de recursos se concentra en torno a las clases medias y altas, produciéndose lo que Herman Deleeck denomina "efecto Mateo" (conjunto de mecanismos específicos que actúan en la sociedad, de tal forma que las clases sociales superiores tienden a beneficiarse más de las ventajas de la política social que las clases inferiores). Así, pues, en la disputa por la asignación de la oferta pública, prevalecen los más fuertes y sus necesidades, como sabemos, no coinciden necesariamente con las de los más débiles, a quienes debemos dirigir nuestros mayores esfuerzos.

A fin de cuentas, en el caso de los Servicios Sociales, nuestra tarea debe orientarse por este camino, hacia la satisfacción de las necesidades humanas, la consecución de una mejora en la calidad de vida, compensando e integrando a los ciudadanos y grupos desfavorecidos en la promoción y universalización del bienestar social (Garcés Ferrer). O bien, satisfaciendo las necesidades humanas insatisfechas, previniendo tales carencias, rehabilitando a los sujetos carenciados, $\mathrm{y}$, si fuese necesario, procurando una acción asistencial de cobertura o apoyo (Casado y Guillén). 
Inevitablemente, la oferta social puede expresar otra dificultad en la satisfacción de las necesidades. También puede generarlas.

Entre las respuestas que ofrece la sociedad para la cobertura de las carencias humanas, hemos conocido la oferta tradicional (protección limitada, consolidadora del status quo), la liberal, la reformista y la marxista. Todas ellas implican diferentes formas de tratar las necesidades humanas. Desde una perspectiva economicista la denominaríamos "demanda social".

Se ha considerado la tercera la más pertinente, aunque la perspectiva crítica se pregunta cómo desde instituciones o realidades emanadas de la heteronomía mayor (el Estado capitalista), puede solucionarse el problema de la pobreza sin consolidar a la misma estructura creadora de desigualdad.

Fuera de esta discusión, fuera de la conceptualización del Trabajo Social como herramienta de cambio, recelosa del poder establecido e, incluso de los mismos Servicios Sociales que éste procura, el gran problema se encuentra en el progresivo triunfo de las propuestas neoliberales. Más aún cuando desde este pensamiento, se considera y, por lo tanto se legitima, la desigualdad. Incluso la entiende como un "mal necesario" que garantiza la continua creación de riqueza y, por lo tanto, la futura posibilidad de una mejora en el bienestar social.

No olvidemos que nuestra Constitución, primer y mejor garante de nuestros derechos, se caracteriza por la profusión de normas programáticas y promotoras. El profesor Lucas Verdú afirma que la Constitución Española de 1978 padece de cierto estrabismo. Esto es: España queda constituida, en un "Estado Social y Democrático de Derecho" (art.1.1.). Por lo tanto, el constituyente observa hacia un lado al Estado Social de Derecho que, respetando el legado liberal fundamental, reconoce la existencia de desigualdades y la necesidad de luchar contra ellas (como así lo concebía Herman Heller: Sozialrechsstaat) y por otro, al Estado Democrático de Derecho, en el que estas desigualdades habrán desaparecido, cosa que, a juicio de insignes juristas, no ha sucedido en ningún país aún.

Todo el Capítulo Tercero de la Constitución ("De los principios rectores de la Política Social y Económica") está plagado de normas programáticas y promotoras que serán satisfechas, pero no se sabe cuándo, pues aún no existe, que yo sepa, ley alguna que las haga efectivas con inmediatez.

No pretendo ahora señalar las grietas y obscuridades de nuestra Constitución, pero sí nos sirve esta reflexión para ilustrar el progresivo desinterés por las libertades negativas, olvidando la pugna por las positivas. Elías Díaz confirma lo que pretendo explicar cuando apunta sobre el triunfo transnacional y hegemónico del neoliberalismo conservador. Tanto en el debate teórico como práctico, en el debate ético y político prevalece, casi exclusivamente, el interés por la libertad negativa, obviando su complementaria. La coordinación entre unas y otras formas de libertad, puede abrirnos el camino hacia una sociedad más justa.

Más aún cuando observamos los retos y fuerzas que pueden debilitar, como así lo hacen, las políticas sociales actuales. Sabemos que la Política Social depende 
de la Política Económica, que la mediatiza y limita. Sabemos también que la construcción del edificio europeo se encamina por los pasos dictados por los eurócratas y la urgencia por alcanzar los trenes europeos de "primera velocidad". Tampoco es menos cierto, a su vez, el progresivo crecimiento de la oferta privada lucrativa en el campo de los Servicios Sociales, con lo que esto puede suponer para una Política Social que se debilita frente a discursos como los de Hayek, Von Mises y Friedman.

Todos estos fenómenos impactan sobre nuestro Sistema Público de Servicios Sociales y, por lo tanto, sobre la profesión de los Trabajadores Sociales. Los nuevos vientos apuestan por el protagonismo del sector privado y del libre mercado. Nadie va a negar la libertad de todo ciudadano a elegir qué tipo de servicios contratar, ni al empresario a crear su propia empresa. Pero estamos hablando de materias referidas a la protección social, materias dotadas de una función social que debe ser privilegiada, pues sobre ellas se fundan y garantizan nuestros derechos, que no pueden ser erosionados por una mala ponderación.

Esta es una tarea bien compleja, por supuesto. En lo que a estas páginas se refiere, mi propuesta se centra en el esfuerzo por emplear mayor rigor en nuestra tarea cotidiana y nuestras investigaciones. Rigor en el empleo de términos y conceptos, rigor que se encuentra en el esfuerzo por unificar criterios, programas y métodos.Incluso si aceptásemos (lo que no tengo excesivamente claro) una perspectiva postmoderna del asunto, estas cuestiones de rigor metodológico, terminológico y, en suma, científico, no serían problema. Más bien, la indefinición misma del Trabajo Social y los Servicios Sociales otorgarían mayores posibilidades para seguir abriéndose camino:

En el Trabajo Social se ha criticado la glorificación del método, el predominio de la cuantificación, y con razón, pero, como todo en la postmodernidad, aquéllo que se critica es de donde se procede. Según la explicación que Howe traza de los rasgos de la postmodernidad en el Trabajo Social, es decir: “...pluralismo y relativismo, reconocimiento de la fragmentación del Trabajo Social; participación de usuarios y desarrollo de la comunicación; poder que mana de la creación de discursos particulares, no de la construcción de un discurso universal limitador; actuación no en la totalidad, sino en cada faceta de la intervención, en la construcción e interacción simbólica de la realidad". Así se podría colegir el hallazgo de una nueva vía para un mejor Trabajo Social. Por lo tanto, la virtud del Trabajo Social postmoderno podría encontrarse en su debilidad, ya que, no adaptándose estrictamente a las exigencias científicas modernas, puede operar con mayor facilidad en un entorno ambiguo, diverso, simultáneo. Pero no podemos creer tal afirmación.

La virtud del pensamiento postmoderno reside, para nosotros, en que introduce más discursos, elimina la visión de la realidad como estructura integrada y perfecta, llena de aristas que no encajan en la sociedad. El pensamiento postmoderno nos deja ver, en todo caso, a la realidad como una configuración sinuosa, no un sistema frío. Pero un pensamiento débil, que niega la racionalidad (de la que, por cierto surge) no garantiza nada, ni siquiera el "fin de la historia". 
A través de la racionalidad, más aún la discursiva, auténtico legado del iluminismo que tanto critica el pensamiento débil, es posible desenmascarar las mismas ideologías que esconden las propias prácticas científicas, postmodernas inclusive, que así pretenden desprenderse de cualquier culpa. No puedo creer que una vuelta al "todo vale" pueda lograr mejorar Servicios Sociales y Trabajo Social. Más de una Escuela y Facultad Universitaria son testigos directos de los problemas surgidos de la falta de criterios comunes, como también es testigo de los esfuerzos empleados en vistas a su unificación.

Nada se construye sobre el desorden, de él sólo se benefician las heteronomías que crean desigualdad. No se trata de creer en un gran, nuevo o antiguo relato emancipador del hombre, sino defender su dignidad, es decir, un desideratum que nos oriente en nuestro trabajo, ya expresado por Juan Luis VIVES hace quinientos años:

"Porque no es justo que en una ciudad rica toleren los magistrados que ciudadano alguno sea maltratado de hambre y de miseria".

A fin de cuentas estamos hablando de justicia social. 
ALEMÁN BRACHO, M.C. y PÉREZ SÁNCHEZ, M.: "Los Servicios Sociales y su relación con otras disciplinas", en ALEMÁN BRACHO, M.C. y GARCÉS FERRER, J., (dirs.) (1996): Administración Social: servicios de bienestar social, Siglo XXI, Madrid.

CASADO, D., en, V Informe Sociológico sobre la situación social en España. Fundación FOESSA, Madrid 1994.

CASADO, D. y GUILLÉN, M.E., (1994): Introducción a los Servicios Sociales Editorial Popular S.A, Madrid.

CHARROALDE VALLÉS, J., "Información y orientación en los centros de servicios sociales -CSS", en Cuadernos de Trabajo Social n ${ }^{\circ} 9$. Escuela Universitaria de Trabajo Social, UCM. Servicio de Publicaciones de la UCM, Madrid 1996.

DELEECK, H., "L'Effet Matthieu” en. Droit Social, Noviembre 1979. Este asunto lo han tratado también PIÑERO Y CHARROALDE "Efecto Mateo y Política Social", en Cuadernos INAS n ${ }^{\circ} 15$ y 16, Julio-Diciembre 1984. DÍAZ, E.,(1990): Los intelectuales y el poder, Centro de Estudios Constitucionales, Madrid .

DURKHEIM, E., (1986): Las reglas del método sociológico, Morata, Madrid . Frente a Vattimo e, incluso, criticando la tentación de la Escuela de Francfort de reducir la modernidad al dominio de la técnica, valga la obra de TOURAINE, A., (1993): Crítica de la Modernidad, Temas de Hoy, Madrid . GARCÉS FERRER, J., (1996): Sistema político y administrativo de los Servicios Sociales, Tirant lo Blanch, Valencia .

GARCÍA LONGORIA Y SERRANO, M. P. (1997): Proyecto Docente. No publicado, Murcia .

HOWE, D., "Modernity, Postmodernity and Social Work", en: The British Journal of Social Work. Vol. 24, n 5. 1994.

KUHN, TH., (1990): La estructura de las Revoluciones Científicas, Fondo de Cultura Económica, Madrid .Sobre los problemas de la ciencia, y sus debilidades, versa la obra de CHALMERS, A.F.,(1987): ¿Qué es esa cosa llamada Ciencia? , Siglo XXI., Madrid . PERDOMO, J.G.,(1990): Servicios Sociales: Leyes Autonómicas, MASS, Madrid.

RUBIOL G., "Servicios Sociales" en VV. AA.: Materiales para un glosario sobre bienestar social. Cáritas Española, Comité Español para el Bienestar Social, Madrid 1986.

SÁNCHEZ RON, J.M., y ECHEVERRÍA, J., "Ciencia moderna y postmoderna", en Boletín Informativo n ${ }^{\circ} 285$, Fundación Juan March, Madrid,Diciembre 1998.

VIVES, J.L., (1992): Del Socorro de los Pobres, HACER, Barcelona . 
El estudio de Deleeck conecta con las investigaciones en torno a la participación política, en el sentido de que aquéllos que más participan son los más oídos, sí, pero entre estos, son los de mayor estatus quienes reciben los beneficios de tal participación. Como puede verse en el estudio clásico de VERBA, S. y NIE, N. H., "Participation and equality: who gets what and how", en KRESEEL, N., (comp.) (1993): Classical and contemporary readings, Parangon House Publishers, New York . 